\title{
Chinese ischemic stroke subclassification
}

\section{S. Gao', Y. J. Wang ${ }^{2}$, A. D. Xü ${ }^{3}$, Y. S. Li" and D. Z. Wang ${ }^{5}$}

\author{
1 Department of Neurology, Peking Union Medical College Hospital, Chinese Academy of Medical Sciences, Peking Union Medical College, Beijing, China \\ 2 Department of Neurology, Beijing Tiantan Hospital, Capital Medical University, Beijing, China \\ ${ }^{3}$ Department of Neurology, The First Affiliated Hospital of Jinan University, Guangzhou, China \\ ${ }^{4}$ Department of Neurology, Renji Hospital, Shanghai Jiaotong University School of Medicine, Shanghai, China \\ 5 Illinois Neurological Institute Stroke Network, OSF Healthcare Systems, Department of Neurology, University of Illinois College of Medicine at Peoria, Peoria, IL, USA
}

\section{Edited by:}

David S. Liebeskind, University of

California Los Angeles, USA

\section{Reviewed by:}

Jose G. Merino, Suburban Hospital, USA

Nerses Sanossian, University of

Southern California, USA

Wengui Yu, University of Texas

Southwestern Medical Center at

Dallas, USA

\section{*Correspondence:}

Y. J. Wang, Department of Neurology,

Beijing Tiantan Hospital, Capital

Medical University, Beijing 100053,

China.

e-mail: yongjunwang111@yahoo.com.cn
Accurate classification of stroke has significant impact on patient care and conduction of stroke clinical trials. The current systems such as TOAST, SSS-TOAST, Korean TOAST, and A-S-C-O have limitations. With the advent of new imaging technology, there is a need to have a more accurate stroke subclassification system. Chinese ischemic stroke subclassification (CISS) system is a new two step system aims at the etiology and then underlying mechanism of a stroke. The first step classify stroke into five categories: large artery atherosclerosis (LAA), including atherosclerosis of aortic arch and intra-/extracranial large arteries, cardiogenic stroke, penetrating artery disease, other etiology, and undetermined etiology. The second step is to further classify the underlying mechanism of ischemic stroke from the intracranial and extracranial LAA into the parent artery (plaque or thrombosis) occluding penetrating artery, artery-to-artery embolism, hypoperfusion/impaired emboli clearance, and multiple mechanisms. Although clinical validation of CISS is being planned, CISS is an innovative system that offers much more detailed information on the pathophysiology of a stroke.

Keywords: ischemic stroke, subclassification, etiology, mechanism, Chinese

\section{INTRODUCTION}

Accurate subclassification of acute ischemic stroke has significant impact on patient care, secondary stroke prevention, and stroke research (conducting clinical trials, epidemiological studies, and genetic studies). Currently the most popular stroke classification system is the TOAST criteria (Adams et al., 1993) which derived from the Harvard Stroke Registry classification (Mohr et al., 1978) and the National Institute of Neurological Disorders and Stroke (NINDS) Stroke Data Bank (Sacco et al., 1989). Recently two newer subclassification systems, the SSS-TOAST (Ay et al., 2005, 2007) and the Korean TOAST (Han et al., 2007) were made available. Both upgraded and optimized diagnostic criteria for the subtypes of atherosclerosis and small artery occlusions. The newly published A-S-C-O classification (Amarenco et al., 2009) is more suitable for secondary stroke prevention, conducting clinical trials, and genetic studies. However, all of these criteria neglected intracranial atheromatous branch disease affecting penetrating arteries. Furthermore, none explored further the underling mechanisms of ischemic strokes caused by large artery atherosclerosis (LAA).

Here is an example of why these criteria have their limitations in the accuracy in discerning the cause of a stroke. In clinical practice, we often encounter patients diagnosed with small vessel disease stroke. Their MRIs often show a singular lacunar infarction that is less than $1.5 \mathrm{~cm}$ in diameter in the territory of the penetrating artery. Vascular imaging show no abnormality in the parenting arteries where this diseased vessel derived from. Clinicians would lump this kind of stroke into either small vessel disease, small artery disease, or small artery occlusion. They would consider that the underlying pathology is lipohylization. Patients would receive antithrombotics as the treatment but often ineffective. This kind of presentation has prompted many clinicians to explore that the true pathology may be is at the origin of these penetrating arteries. Therefore, the stroke symptoms would be related to local hemodynamic or perfusion deficiency.

With the advent of imaging technology such as 64-slice CT scanners, contrast enhanced MRA (CEMRA), CT angiography (CTA), CT and MRI perfusion, high resolution MRI/MRA (HR-MRI/ MRA), TCD-microembolic signal (TCD-MES) detection, transesophageal echocardiography (TEE), and MRI of the heart, more previously non-visible underlying pathology and ambiguous lesions can now be clearly visualized. Utilizing the information from these new imaging studies, Chinese ischemic stroke subclassification (CISS) attempts to subclassify a stroke with greater accuracy. It is an improved approach to ischemic stroke subtyping.

\section{USING CISS, THE TWO STEPS (FIGURES 1-3) THE FIRST STEP, DEFINING FIVE CATEGORIES OF STROKE TYPES Large artery atherosclerosis (LAA)}

In CISS, LAA includes atherosclerosis of aortic arch and intra-/ extracranial large arteries.

\section{Aortic arch atherosclerosis}

(1) Acute multiple infarcts, especially involving bilateral anterior and/or anterior and posterior circulations; (2) No evidence of atherosclerosis of relevant intracranial or extracranial large arteries (vulnerable plaques or stenosis $\geq 50 \%$ or occlusion); (3) No evidence of potential cause of cardiogenic stroke (CS); (4) No evidence of other etiologies that can cause multifocal acute ischemic infarcts such as vasculitidies, hemostatic disturbances, and tumorous 


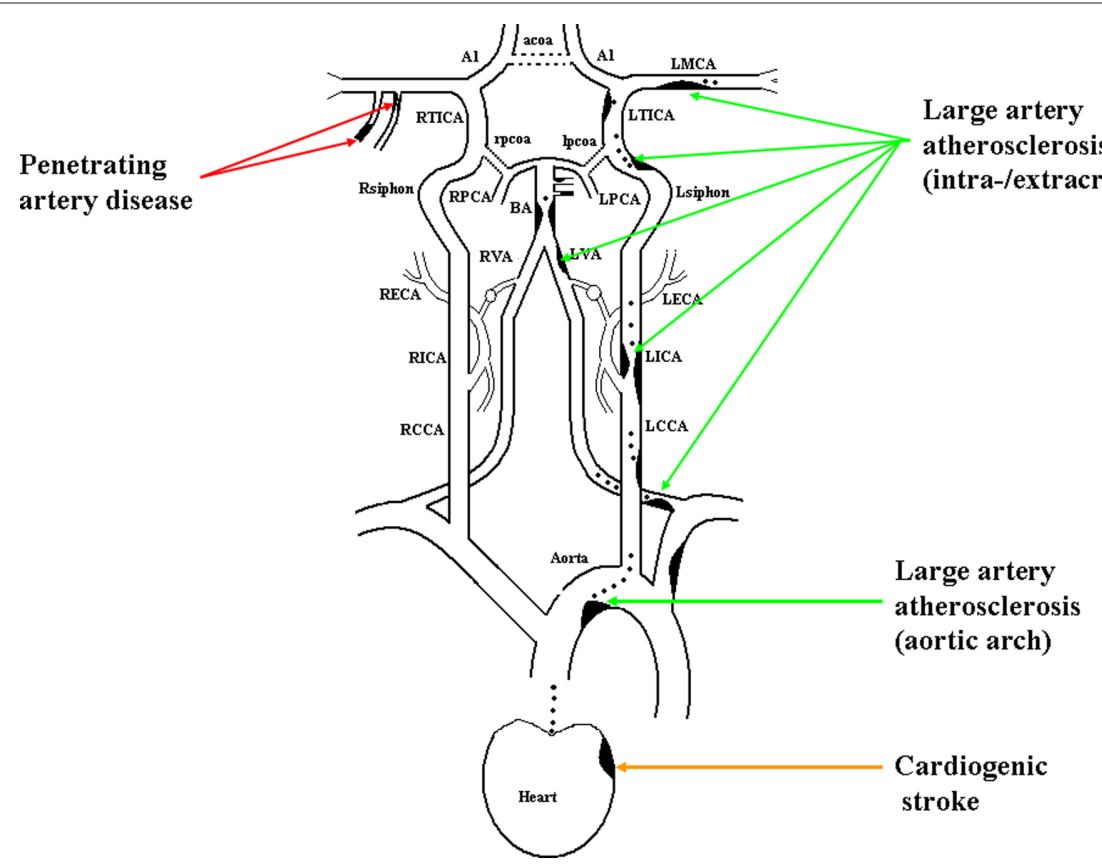

FIGURE 1 | Illustration of large artery atherosclerosis, cardiogenic stroke, and penetrating artery disease.

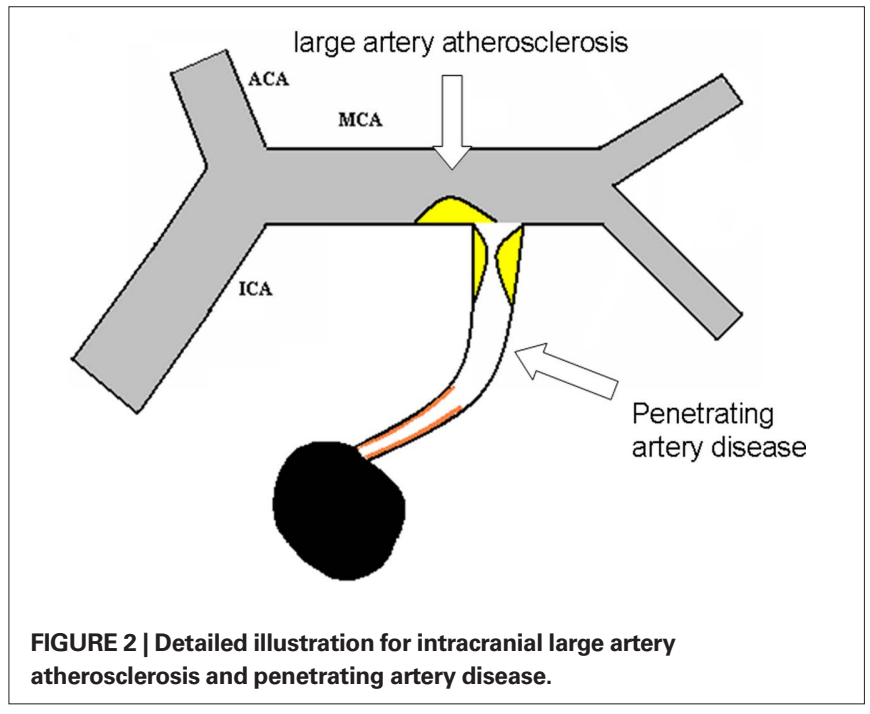

embolism; (5) Evidence of significant aortic arch atherosclerosis (aortic plaques $>4 \mathrm{~mm}$ and/or aortic thrombi, detected by HR-MRI/ MRA and/or TEE; Reynolds et al., 2003; Harloff et al., 2008).

\section{Intra- and extracranial large arteries atherosclerosis}

(1) Any distribution of acute infarcts (except isolated infarct in the territory of one penetrating artery), with evidence of atherosclerosis involving intracranial or extracranial large arteries (vulnerable plaques or stenosis $\geq 50 \%$ ) that supply the area of infarction; (2) Concerning isolated penetrating artery territory infarct, the following circumstance should also be included in LAA: with evidence of atherosclerotic plaque (detected by HR-MRI) or any degree of stenosis in the parent artery (detected by TCD, MRA, CTA, or DSA); (3) No evidence of potential cardiac-origin embolic cause; (4) Other possible causes have also been excluded.

\section{Cardiogenic stroke}

(1) Acute multiple infarcts, especially involving bilateral anterior and/or anterior and posterior circulations (including cortical infarcts) that have occurred closely in time; (2) No evidence of atherosclerosis on relevant intracranial or extracranial large arteries (vulnerable plaques or stenosis $\geq 50 \%$ or occlusion); (3) No evidence of other etiologies that can cause multifocal acute ischemic infarcts such as vasculitidies, hemostatic disturbances, and tumorous embolism; (4) Evidence of cardiac disease that has a potential for embolism; (5) If the possibility of aortic arch atherosclerosis has been excluded, CS is definite. Otherwise, the category should be possible CS.

The potential lesions included under this category are: mitral stenosis, prosthetic heart valve, myocardial infarction within the past 4 weeks, mural thrombus in the left cavities, left ventricular aneurysm, any documented history of permanent or transient atrial fibrillation or flutter with or without spontaneous echo contrast or left atrial thrombus, sick sinus syndrome, dilated cardiomyopathy, ejection fraction $<35 \%$, endocarditis, intracardiac mass, PFO plus in situ thrombosis, PFO plus concomitant PE, or DVT preceding the brain infarction (Amarenco et al., 2009).

\section{Penetrating artery disease}

Acute isolated infarct in the territory of one penetrating artery caused by atherosclerosis at the proximal segment of the penetrating arteries or lipohyalinotic degeneration of arterioles is called penetrating artery disease (PAD). 


\section{Overview of CISS}

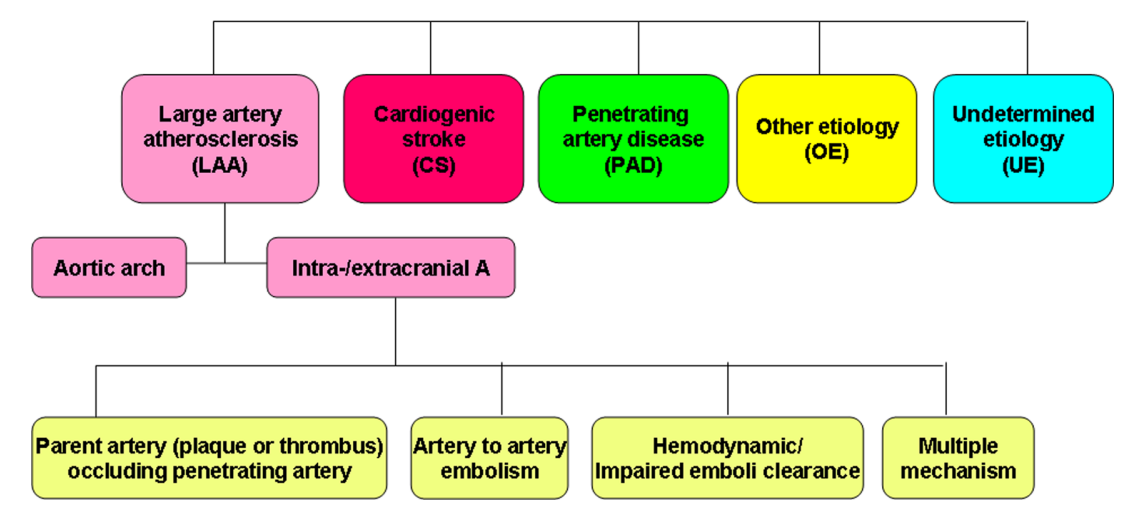

FIGURE 3 | Overview of CISS.

(1) Acute isolated infarct in clinically relevant territory of one penetrating artery, regardless of the size of infarct; (2) No evidence of atherosclerotic plaque (detected by HR-MRI) or any degree of stenosis in the parent artery (detected by TCD, MRA, CTA, or DSA). (3) With evidence of vulnerable plaques or stenosis $\geq 50 \%$ in ipsilateral proximal intracranial or extracranial large arteries, isolated penetrating artery infarct is classified in undetermined etiology (UE; multiple etiology); (4) With evidence of cardiac disease that has a potential for embolism, isolated penetrating infarct is classified in UE (multiple etiology); (5) Other possible causes has been excluded.

\section{Other etiologies}

Evidence of other specific diseases (e.g., vascular related disease, infective disorder, inherited disease, hematological system disorder, vasculitis), that are relevant to the index stroke and can be demonstrated by blood tests, cerebrospinal fluid (CSF) tests, and vascular imaging. The possibility of LAA or CS has been excluded.

\section{Undetermined etiology}

No evidence of any specific potential etiology that is clinically relevant to the index stroke.

Multiple: Evidence of more than one potential cause, but difficult to determine which was the relevant cause of the index stroke.

Unknown: No determined cause is responsible for the index stroke unless more investigations would be performed.

Inadequate evaluation: Routine assessments of intracranial and extracranial arteries or heart are not completed, which makes the etiology undetermined.

\section{THE SECOND STEP, DEFINING THE UNDERLYING MECHANISM FOR ISCHEMIC STROKE OF INTRACRANIAL OR EXTRACRANIAL LARGE ARTERY ATHEROSCLEROSIS (FIGURE 3)}

In the CISS system, the underlying mechanisms of ischemic stroke caused by intracranial or extracranial LAA are further defined as the parent artery (plaque or thrombus) occluding penetrating artery, artery-to-artery embolism, hypoperfusion/impaired emboli clearance, and multiple mechanism.
1. Parent artery (plaque or thrombus) occluding penetrating artery: Isolated acute infarct in penetrating artery territory, the parent artery has evidence of plaque or any degree of stenosis. For example, an isolated acute infarct located in the basal ganglia, and no other acute infarct in the territory supplied by the ipsilateral MCA; or an isolated acute infarct located in the pons, and no other acute infarct in the territory supplied by the basilar artery. The acute isolated infarct is posited to be caused by the plaque of the parent artery protruding and subsequently occluding blood flow to the penetrating artery.

2. Artery-to-artery embolism: Imaging shows small cortical infarcts or a single territory infarct in the area supplied by the relevant intracranial or extracranial artery atherosclerosis. No borderzone infarct related to this diseased artery. This diagnosis is confirmed if the infarcts are multiple, or the single infarct is accompanied by TCD-MES on TCD. However, a single cortical or territorial infarct without obtaining MES can also be diagnosed as artery-to-artery embolism.

3. Hypoperfusion/impaired emboli clearance: the acute infarcts occur solely in the borderzone area. No acute cortical or territory infarcts related to this diseased artery. The degree of stenosis of the clinically relevant intracranial or extracranial artery is usually $>70 \%$ with or without evidence of hypoperfusion or poor collateral compensation in the related region of perfusion.

4. Multiple mechanism: two or more underlying mechanisms mentioned above combined together.

\section{ILLUSTRATIONS OF CISS}

Comparing to other classification systems, CISS has the following major differences: (1) aortic arch atherosclerosis belongs to LAA. Given that the intrinsic lesion is atherosclerosis, it is more reasonable to classify aortic arch atherosclerosis into LAA (Amarenco et al., 2009). (2) In acute isolated penetrating artery territory infarcts, they would be classified into LAA if there was atherosclerotic plaque or stenosis of the parent artery regardless of the presence of any plaques or stenosis. (3) PAD is a new concept. Excluding other diseases, acute isolated penetrating artery territory infarcts are 
considered to be caused by the penetrating artery lesions. Pathology has shown that atheromatous disease at the proximal segment of the penetrating arteries mainly lead to symptomatic infarcts, while fibrohyalinosis of arterioles is mainly associated with asymptomatic lacunes or diffuse white matter hyperintensities (Fisher, 1969, 1979; Fisher and Caplan, 1977).

Caplan (1989) noted that intracranial branch atheromatous disease was a neglected, understudied, and underused concept. Unfortunately, in the past 20 years, it has been rare to find vascular pathology studies that trace the entire penetrating arteries and their parent arteries. Researchers either studied the parent artery (leading to the finding of atherosclerosis of parent artery) or more distal segment of the penetrating artery and the arteriole (leading to the finding of arteriolar fibrohyalinosis; Lammie et al., 1979; Chen et al., 2008; Klein et al., 2010). Fibrohyalinosis of small arteries and arterioles causes symptomatic acute penetrating artery infarcts less often than atheromatous branch disease. Therefore, the current opinion that symptomatic penetrating artery territory lesions are equivalent to intrinsic small vessel disease is inappropriate.

Even if atherosclerotic lesions involving the proximal segment of the penetrating arteries was not considered the main cause of symptomatic penetrating artery territory infarcts, it should be taken into account as well as arteriolar fibrohyalinosis. Diffuse white matter hyperintensities can reflect arteriolar fibrohyalinosis, but atherosclerotic lesions and fibrohyalinosis often coexist (Fisher, 1965), and current imaging technology can not show the penetrating artery wall directly. Therefore, clinically it is difficult to distinguish between the two. With further improvements in imaging, there could develop further PAD subclassifications. In addition, the introduction of the concept of PAD not only avoids confusion with intrinsic small vessel disease, but also avoids confusion with the "lacunar" concept.

In LAA, several outcomes of atherosclerotic stenosis or occlusion of the carotid artery may occur: (1) There is no infarct or ischemic symptoms because the fragments of plaques or thrombi do not dislodge and there is a well-compensated Willis' circle. (2) The fragments of plaques or thrombi do not dislodge, but borderzone infarction may occur if there is a poorly compensated Willis' circle. Cerebral perfusion may be compromised due to hypoperfusion events such as sudden drop in blood pressure or in other systemic hypoperfusion events (Momjian-Mayor and Baron, 2005). (3) If the fragments of plaques or thrombi dislodge and travel to distal branches, this may lead to a mechanism of artery-to-artery embolism or impaired emboli clearance depending on the location of the infarcts (Fisher and Caplan, 1977; Derdeyn et al., 2001; Chaves et al., 2003). The mechanism of vertebral artery infarction is similar to that of the extracranial carotid artery.

For intracranial large arteries (MCA's), thrombosis on atheroma will make the stenosis more severe and may lead to complete occlusion of the artery. There are several processes and outcomes of atherothrombotic stenosis or occlusion of the MCA (Wong

\section{REFERENCES}

Adams, H. P.Jr., Bendixen, B. H., Kappelle, L. J., Biller, J., Love, B. B., Gordon, D. L., and Marsh, E. E. III. (1993). Classification of subtype of acute ischemic stroke. Definitions for use in a multicenter clinical trial. TOAST. Trial of Org 10172 in acute stroke treatment. Stroke 24, 35-41.

Amarenco, P., Bogousslavsky, J., Caplan, L. R., Donnan, G. A., and Hennerici, M. G. (2009). New approach to stroke

et al., 2002; Bang et al., 2004; Lee et al., 2005; Caplan et al., 2006): (1) The fragments of plaques or thrombi do not dislodge, the penetrating artery is not blocked by plaques or thrombi, there is a well-compensated pia mater collateral circulation and the newly generated collateral arteries can supply the territory of the penetrating arteries. This MCA territory can endure long-term ischemia and there will be no infarcts in this region even after the MCA is completely occluded. (2) The fragments of plaques or thrombi do not dislodge, the penetrating artery is not blocked by atherosclerosis and there is poorly established collateral circulation. Borderzone infarction may occur if cerebral perfusion is compromised due to hypoperfusion. This mechanism is called hypoperfusion. (3) If the orifice of one or more penetrating arteries are blocked by plaque or thrombi, infarction will occur in the regions of the penetrator. This mechanism is called parent artery occluding penetrating artery. (4) If the fragments of plaques or thrombi are dislodged and travel to distal branches, this mechanism is labeled artery-to-artery embolism or impaired emboli clearance based on the location of the infarcts. The underlying mechanism of infarction related to the basilar artery is similar to that of MCA.

The mechanism of hypoperfusion refers to borderzone infarction from compromised hemodynamics. The mechanism of impaired emboli clearance means that the borderzone infarct is posited to result from impaired clearance of emboli due to both hypoperfusion and embolism. Actually, it is difficult to completely separate these two mechanisms. For a patient with ICA or MCA stenosis (>70\%), develop a borderzone infarct and TCD also detects MES, then the underlying mechanism would be impaired emboli clearance. On the other hand, we cannot exclude the existence of impaired emboli clearance mechanism even though there is no MES detected by TCD. Therefore, it would be more appropriate to combine the two mechanisms together.

\section{CONCLUSION}

Chinese ischemic stroke subclassification introduces a new way of subclassifying acute ischemic stroke that takes into consideration of both etiological and pathophysiological information. In CISS, aortic arch atherosclerosis is classified into LAA, and a new subclassification of PAD has been created. The underling mechanism of ischemic stroke from the intracranial and extracranial LAA has been subclassified into four categories: the parent artery (plaque or thrombus) occluding penetrating artery, artery-to-artery embolism, hypoperfusion/impaired emboli clearance, and multiple mechanisms. CISS is an improved and more rational way of stroke subtyping. It has deepened our understanding into the pathophysiology of stroke.

\section{ACKNOWLEDGMENT}

The authors would like to express their sincere gratitude to Dr. Louis R. Caplan for his time, expertise and advise on editing, and finalizing this manuscript.

subtyping: the A-S-C-O (phenotypic) classification of stroke. Cerebrovasc. Dis. 27, 502-508.

Ay, H., Benner, T., Arsava, E. M., Furie, K.

L., Singhal, A. B., Jensen, M. B., Ayata,

C., Towfighi, A., Smith, E. E., Chong,
J. Y., Koroshetz, W. J., and Sorensen, A. G. (2007). A computerized algorithm for etiologic classification of ischemic stroke: the causative classification of stroke system. Stroke 38, 2979-2984. 
Ay, H., Furie, K. L., Singhal, A., Smith, W. S., Sorensen, A. G., and Koroshetz, W. J. (2005). An evidence-based causative classification system for acute ischemic stroke. Ann. Neurol. 58, 688-697.

Bang, O. Y., Joo, S. Y., Lee, P. H., Joo, U. S., Lee, J. H., Joo, I. S., and Huh, K. (2004). The course of patients with lacunar infarcts and a parent arterial lesion: similarities to large artery vs small artery disease. Arch. Neurol. 61, 514-519.

Caplan, L. R. (1989). intracranial branch atheromatous disease: a neglected, understudied, and underused concept. Neurology 39, 1246-1250.

Caplan, L. R., Wong, K. S., Gao, S., and Hennerici, M. G. (2006). Is hypoperfusion an important cause of strokes? If so, how. Cerebrovasc. Dis. 21, 145-153.

Chaves, C. J., Silver, B., Schlaug, G., Caplan, L. R., and Warach, S. (2003). Diffusion- and perfusion-weighted MRI patterns in borderzone infarcts. Stroke 31, 1091-1096.

Chen, X. Y., Wong, K. S., Lam, W. W. M., Zhao, H.-L., and Ng, H. K. (2008). Middle cerebral artery atherosclerosis: histological comparison between plaques associated with and not associated with infarct in a postmortem study. Cerebrovasc. Dis. 25, 74-80.

Derdeyn, C. P., Khosla, A., Videen, T. O., Fritsch, S. M., Carpenter, D. L., Grubb,
R. L. Jr., and Powers, W. J. (2001). Sever hemodynamic impairment and border zone-region infarction. Radiology 220, 195-201.

Fisher, C. M. (1965). Lacunes: small, deep cerebral infarcts. Neurology 15, 774-784.

Fisher, C. M. (1969). The arterial lesions underlying lacunes. Acta Neuropathol. 12, 1-15.

Fisher, C. M. (1979). Capsular infarcts. Arch. Neurol. 36, 65-73.

Fisher, C. M., and Caplan, L. R. (1977). Bilateral occlusion of basilar artery branches. J. Neurol. Neurosurg. Psychiatr. 40, 1182-1189.

Han, S. W., Kim, S. H., Lee, J. Y., Chu, C. K., Yang, J. H., Shin, H. Y., Nam, H. S., Lee, B. I., and Heo, J. H. (2007). A new subtype classification of ischemic stroke based on treatment and etiologic mechanism. Eur. Neurol. 57, 96-102.

Harloff, A., Dudler, P., Frydrychowicz, A., Strecker, C., Stroh, A. L., Geibel, A., Weiller, C., Hetzel, A., Hennig, J., and Markl, M. (2008). Reliability of aortic MRI at 3 Tesla in patients with acute cryptogenic stroke. J. Neurol. Neurosurg. Psychiatr. 79, 540-546.

Klein, I. F., Labreuche, J., Lavallée, P. C., Mazighi, M., Duyckaerts, C., Hauw, J. J., and Amarenco, P. (2010). Is moderate atherosclerotic stenosis in the middle cerebral artery a cause of or a coincidental finding in ischemic stroke? Cerebrovasc. Dis. 29, 140-145. Lammie, G.A., Brannan, F., Slattery, J., and Warlow, C. (1979). Nonhypertensive cerebral small-vessel disease an autopsy study. Stroke 28, 2222-2229.

Lee, D. K., Kim, J. S., Kwon, S. U., Yoo, S. H., and Kang, D. W. (2005). Lesion patterns and stroke mechanism in atherosclerotic middle cerebral artery disease: early diffusion-weighted imaging study. Stroke 36, 2583-2588.

Mohr, J. P., Caplan, L. R., Melski, J. W., Goldstein, R. J., Duncan, G. W., Kistler, J. P., Pessin, M. S., and Bleich, H. L. (1978). The Harvard Cooperative Stroke Registry: a prospective registry. Neurology 28, 754-762.

Momjian-Mayor, I., and Baron, J. C. (2005). The pathophysiology of watershed infarction in internal carotid artery disease: reviews of cerebral perfusion studies. Stroke 36, 567-557.

Reynolds, H. R., Tunick, P. A., and Kronzon, I. (2003). Role of transesophageal echocardiography in the evaluation of patients with stroke. Curr. Opin. Cardiol. 18, 340-345.

Sacco, R. L., Ellenberg, J. H., Mohr, J. P., Tatemichi, T. K., Hier, D. B., Price, T. R., and Wolf, P. A. (1989). Infarcts of undetermined cause: the NINCDS Stroke Data Bank. Ann. Neurol. 25, 382-390.
Wong, K. S., Gao, S., Chan, Y. L., Hansberg, T., Lam, W. W., Droste, D. W., Kay, R., and Ringelstein, E. B. (2002). Mechanisms of acute cerebral infarctions in patients with middle cerebral artery stenosis: a diffusion-weighted imaging and microemboli monitoring study. Ann. Neurol. 52, 74-81.

Conflict of Interest Statement: The authors declare that the research was conducted in the absence of any commercial or financial relationships that could be construed as a potential conflict of interest.

Received: 02 October 2010; accepted: 31 January 2011; published online: 15 February 2011.

Citation: Gao S, Wang YJ, Xu AD, Li YS and Wang DZ (2011) Chinese ischemic stroke subclassification. Front. Neur. 2:6. doi: 10.3389/fneur.2011.00006

This article was submitted to Frontiers in Stroke, a specialty of Frontiers in Neurology.

Copyright (C) 2011 Gao, Wang, Xu, Li and Wang. This is an open-access article subject to an exclusive license agreement between the authors and Frontiers Media $S A$, which permits unrestricted use, distribution, and reproduction in any medium, provided the original authors and source are credited. 